\title{
C-Reactive Protein and Frailty in the Elderly: A Literature Review
}

\author{
Dimitrios Velissaris $^{\mathrm{a}, \mathrm{g}}$, Nikolaos Pantzaris ${ }^{\mathrm{b}}$, Ioanna Koniari ${ }^{\mathrm{c}}$, Nikolaos Koutsogiannis ${ }^{\mathrm{c}}$, \\ Vasilios Karamouzos $^{\mathrm{d}}$, Ioanna Kotroni ${ }^{\mathrm{e}}$, Anastasia Skroumpelou ${ }^{\mathrm{f}}$, John Ellul ${ }^{\mathrm{f}}$
}

\begin{abstract}
Chronic inflammation is a well-established background process in many age-related diseases. Many recent studies investigate the use of various inflammatory biomarkers such as C-reactive protein (CRP), interleukin-6, and interleukin-1 as predictors of physical and cognitive performance among elders. The phenotype of frailty has also been associated with underlying inflammatory mechanisms. The aim of this article was to review the literature referring to the correlation of CRP serum levels and frailty in older individuals. We tried to identify all relevant publications regarding the relation of CRP as an index of frailty in the elderly and its potential use. Although many studies in the recent medical literature positively associate serum CRP levels and frailty in older individuals, some do not, and some raise some interesting questions and set the basis for future studies. The association of CRP and frailty in elder patients should be considered when clinicians interpret inflammatory biomarkers in various clinical settings in such patients. Well-designed, prospective clinical trials are warranted to better assess the role and pathophysiology of frailty in the elderly and its mechanisms as also the exact role of CRP as an inflammatory marker and as a prognostic index in this syndrome.
\end{abstract}

Keywords: C-reactive protein; Elderly; Frailty

\section{Introduction}

Chronic low-grade inflammation (inflammaging) in the elder population is considered a risk factor for the development of aging-related diseases and frailty. Geriatric frailty is associated with increased inflammatory activity as increased levels of several biomarkers like TNF-a, interleukin-6 (IL-6), cytokine

Manuscript accepted for publication March 24, 2017

anternal Medicine Department, University Hospital of Patras, Greece

bUniversity Hospital of Patras, Greece

'Department of Cardiology, University Hospital of Patras, Greece

dintensive Care Unit, University Hospital of Patras, Greece

${ }^{\mathrm{e}}$ Coronary Care Unit, University Hospital of Patras, Greece

fDepartment of Neurology, University Hospital of Patras, Greece

${ }^{g}$ Corresponding Author: Dimitrios Velissaris, Internal Medicine Department,

University Hospital of Patras, Greece. Email: dimitrisvelissaris@yahoo.com

doi: https://doi.org/10.14740/jocmr2959w antagonists and acute phase proteins are identified in the serum of frail older individuals. C-reactive protein (CRP) is playing a key role in several disease processes, and elevated serum CRP levels have been identified to accompany increased vulnerability for disease and mortality in older patients. The aim of this review was to search for the relation between the commonly used inflammatory marker CRP and the risk of incidence of physical frailty in older individuals as extracted from the published literature. We conducted a PubMed search using the combinations of the terms "CRP", "C-reactive protein" AND "frailty", "frail", "elders" or "elderly". All identified manuscripts were considered for inclusion in this review. We also reviewed the bibliographies of all extracted manuscripts attempting to identify additional relevant publications. The database search, as well as the review of the references of the relevant publications, resulted in a total of 29 studies conducted between 2004 and 2016 which discussed and investigated the relationship between CRP and frailty in elderly individuals. The studies are presented along with their main findings in Table 1 [1-29].

\section{Frailty in the Elderly}

Frailty is defined as the syndrome characterized by a reduced ability of individuals to re-establish homeostasis in response to stress. The phenotype of physical frailty consists of weight loss, slowness, weakness, exhaustion, and a low physical activity level. The causes of frailty seem to be complex, as genetic, biological, physical, social and environmental factors are involved in its pathogenesis. The mechanisms of the syndrome are multifactorial, as inadequate nutrition, endocrine, and immune system dysfunctions are involved in its development. Additionally, disadvantaged socioeconomic conditions and low cultural levels seem to be significantly related to this clinical entity [30,31].

The clinical syndrome of frailty in the elderly consists of several pathologies and is characterized by low physical activity, weakness, exhaustion, and a global impairment of physiological reserves of several organ systems. The incidence of geriatric frailty is around $20-30 \%$ of the population over 75 years and increases with advancing age. Age and chronic disease-related activation of inflammation, as also neuroendocrine dysregulation and metabolic changes lead to physiological and clinical frailty. The fact that frailty in the elderly is related to long-term adverse health-related outcomes like disability, dependency, hospitalization and mortality is of great importance. 
Table 1. Summary of Studies

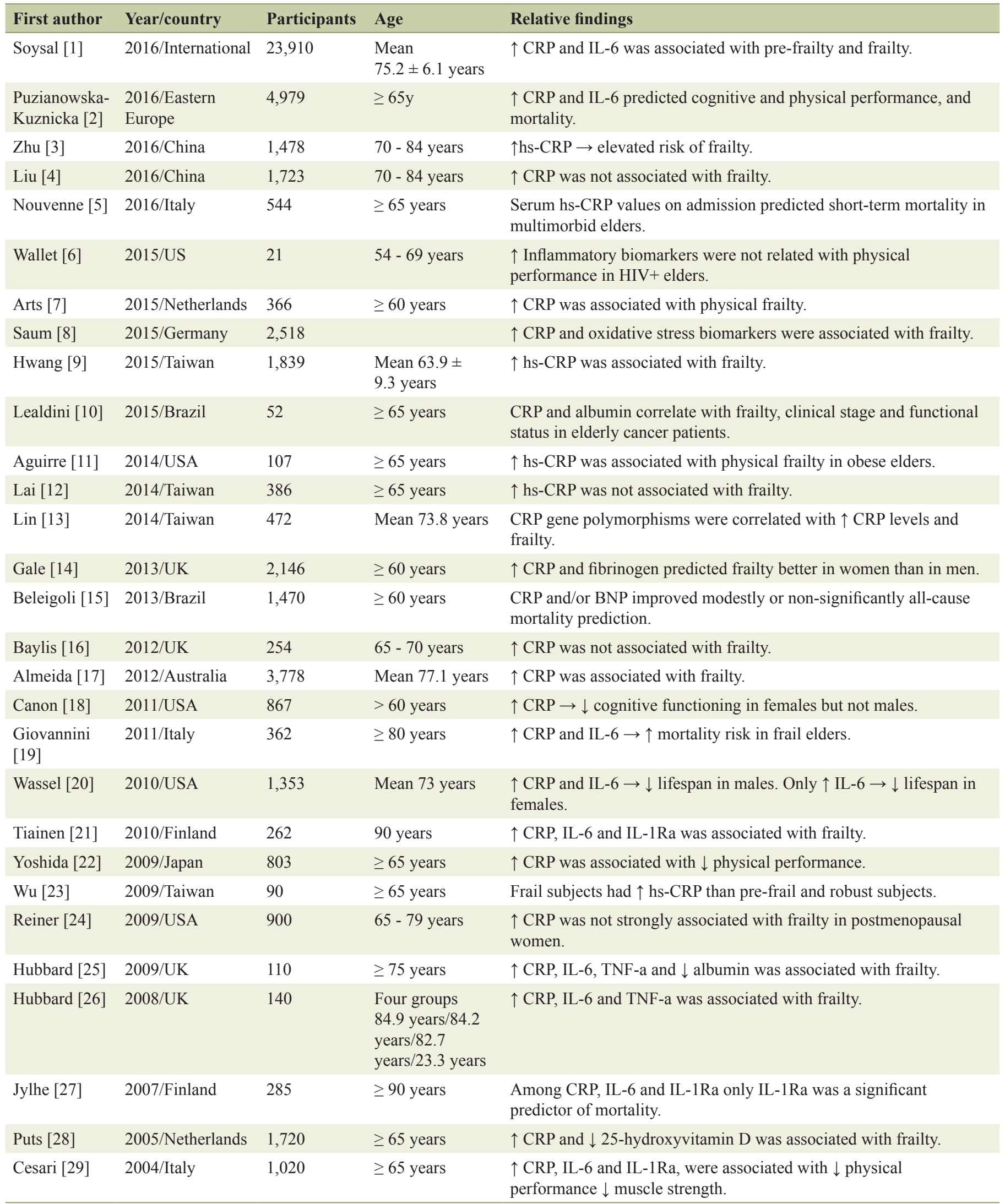




\section{Frailty and Inflammatory Biomarkers}

The chronic activation of the inflammatory response, called inflammaging, is considered a key component for the development of frailty in older individuals. Low-grade chronic inflammation and oxidative stress, mediated partly from the superoxide anion overproduction by NADPH oxidase, consists part of the underlying pathology. Several biomarkers, like erythrocyte sedimentation rate, CRP, white blood cell and lymphocyte counts, iron, albumin, cholesterol and other, are associated with a higher severity grade of the multidimensional prognostic index (MPI) and mortality [32]. Many studies show that the clinical phenotype of frailty is associated with pathologic levels of laboratory markers suggesting as possible pathogenetic mechanisms hormonal dysregulation, immuno-aging, pro-coagulation and pro-inflammatory abnormalities [33].

\section{Frailty and CRP}

A useful approach to assess frailty in clinical settings is the use of biomarkers, thus making feasible and accurate the assessment of frailty by clinicians. Standard clinical tests and observations associated with inflammation are hypoalbuminemia, erythropoietin resistance, decreased iron saturation accompanied by high ferritin, physical frailty, low serum creatinine, reduced total and LDL-cholesterol, and increased CRP. CRP is an important inflammatory biomarker linked to many diseases.

Frailty in the elderly is correlated with higher serum levels of inflammatory biomarkers like IL-6, CRP and TNF-a, which are inversely correlated with poor physical activity, muscle weakness and increased disability $[1-3,7-9,17,21-23,25,26$, 29, 34-37]. Elevated CRP levels are also associated with increased risk of mortality in frail older subjects $[2,5,19]$, but they are not always a significant mortality predictor [15, 27]. Wassel et al in a prospective study with 1,353 participants in 2010 concluded that higher serum CRP levels predicted a reduced survival time only among males [20].

Serum CRP levels were also associated with incident frailty in more specific subgroups, such as poor functional status and more advanced clinical stage in older patients with cancer [10] and impaired physical performance in obese older adults [11]. However, Reiner et al in a 2009 prospective study concluded that there is little evidence associating CRP levels and frailty among postmenopausal women [24]. Among the studies reviewed, two of them showed significant correlations between incident frailty, cognitive functions, and serum CRP concentrations only among women over 60 years old but not among men $[14,18]$.

Studies investigating specific CRP gene polymorphisms and possible associations with frailty among older individuals were also identified. Statistically significant correlations were found between hand grip, as a frailty index, and three out of the five single nucleotide polymorphisms (SNPs) in the CRP gene as well as the haplotype C-C-C-C-C, studied by Lin et al in 2014 in a study involving 472 elderly subjects [13]. Another CRP polymorphism found to be linked with increased odds of frailty was the CRP1846G $>$ A [17]. Two other common CRP gene polymorphisms, rs1205 and rs3093059, were found to be significantly associated with serum CRP levels but not with frailty in an elderly Chinese population [4].

In many specific populations, like patients with heart failure, studies show that there are specific relations between inflammatory markers and physical function. A higher frailty phenotype score was correlated with lower 25-hydroxy vitamin D and higher high sensitivity CRP serum levels in patients with cardiac failure [38]. The acute phase reactant CRP increases with age and increased plasma levels of it have been identified as a biological component of frailty, defining elevated vulnerability for diseases and mortality with aging. Inflammatory markers seem to play a major role and are closely related to frailty as this is confirmed in the very old $(85+)$ by the Newcastle $85+$ study, previously established in younger old populations [39].

Literature review shows that even mild increases in CRP plasma levels are associated with an increased risk of sarcopenia, cardiovascular diseases, disability and cognitive decline in individuals over 65 years old [40]. Also, in a study by Yano et al, it was shown that after assessment of plasma pentraxin-3 and high sensitive CRP for the early detection of cognitive decline in the elderlies, both parameters were significantly associated with the cognitive function calculated by the minimental state examination score [41].

Among the studies presented and summarized in Table 1, two large cohort studies support the direct association of serum CRP levels with frailty. The Cardiovascular Health Study (CHS) by Walston et al showed a significant relation of elevated CRP levels with frailty after excluding cardiovascular disease and diabetes and adjusting for basic demographic characteristics, and the published data from the Longitudinal Aging Study Amsterdam (LASA) [28, 42]. Although a large number of studies show an association between serum CRP levels and the presence of frailty in older patients, few others do not support these findings, concluding that no significant associations are found $[12,16]$.

\section{Potential Clinical Uses}

The assessment of inflammatory markers in the setting of frailty in elderlies may represent a useful screening test and a potential target for further intervention. Of course, having a high concentration of more than one inflammatory marker may be more strongly predictive of incident frailty than a high concentration of only one. Measurement of serum CRP levels is a widely accepted screening test that handles in daily clinical practice. Clinicians are initially using it as a tool to diagnose infections or clinical conditions closely associated with underlying inflammatory mechanisms. As the aging immune system is characterized by a low-grade, chronic systemic inflammatory state, markedly elevated inflammatory molecules, such as CRP could be evaluated in an emergency or long-term basis as part of the assessment in the specific elderly population. CRP may play the role of a useful biomarker for the detection of frailty in elderly individuals as most of the published studies until now are showing a close relation with this clinical entity. 
Clinicians assessing older patients should always consider in their differential diagnosis that elevated levels of serum CRP could be part of an underlying chronic inflammatory process related to the syndrome of frailty in the elderly.

Some meaningful and helpful information are revealed from this review. A large number of studies, referring to different ethnic populations are mentioning the correlation of increased serum CRP levels with increased incidence of frailty in elder individuals. Studies presenting sex-specific differences between frailty and serum CRP levels were also identified [14, $18,24]$. For extremely old populations like the centenarians, there is still a lack of large studies assessing further the role of CRP in frailty. Also, the fact that there are few studies not replicating these findings, as well as studies implicating other biomarkers, inflammatory or not, with the frailty syndrome underlies the need for a better understanding of its pathophysiology. To understand better these pathophysiological pathways leading to the onset of frailty and disability in the elderly, clinical trials are needed assessing healthy elderly populations with increased serum inflammatory markers. Finally, a question should always be raised in clinicians whether elevated serum CRP levels should be taken into account as an index of acute inflammation or as part of the inflammaging (chronic inflammation) process.

\section{Conclusions}

Frailty in the elderly appears in close association with chronic inflammation, and most of the published studies show a correlation between CRP and this clinical entity in older individuals. Further research is needed to investigate the frailty-related pathologies as frailty seems to have substantial health and economic implications, and the screening of frailty using inflammatory markers should be a future debate. As CRP has already been integrated part of the routinely measured biochemical panel for the investigation of many clinical entities, a potential role of such biomarkers and whether they can serve as indexes of vulnerability to age-related diseases is warranted.

\section{Author Contributions}

DV did literature search, wrote and edited the paper. NP collected data and wrote the paper. IK did literature search. NK collected data. VK did literature search. IK did literature search. AS collected data. JE wrote and edited the paper.

\section{Conflicts of Interest}

All authors state that they do not have any conflicts of interest to report.

\section{References}

1. Soysal P, Stubbs B, Lucato P, Luchini C, Solmi M, Peluso
$\mathrm{R}$, Sergi $\mathrm{G}$, et al. Inflammation and frailty in the elderly: A systematic review and meta-analysis. Ageing Res Rev. 2016;31:1-8.

2. Puzianowska-Kuznicka M, Owczarz M, WieczorowskaTobis K, Nadrowski P, Chudek J, Slusarczyk P, Skalska A, et al. Interleukin- 6 and C-reactive protein, successful aging, and mortality: the PolSenior study. Immun Ageing. 2016;13:21.

3. Zhu Y, Liu Z, Wang Y, Wang Z, Shi J, Xie X, Jin L, et al. C-reactive protein, frailty and overnight hospital admission in elderly individuals: A population-based study. Arch Gerontol Geriatr. 2016;64:1-5.

4. Liu ZY, Wang ZD, Li LZ, Chu XF, Zhu YS, Shi JM, Xie XJ, et al. Association of CRP gene polymorphisms with CRP levels, frailty and co-morbidity in an elderly Chinese population: results from RuLAS. Age Ageing. 2016;45(3):360-365.

5. Nouvenne A, Ticinesi A, Lauretani F, Maggio M, Lippi G, Prati B, Borghi L, et al. The Prognostic Value of Highsensitivity C-reactive Protein and Prealbumin for Shortterm Mortality in Acutely Hospitalized Multimorbid Elderly Patients: A Prospective Cohort Study. J Nutr Health Aging. 2016;20(4):462-468.

6. Wallet MA, Buford TW, Joseph AM, Sankuratri M, Leeuwenburgh C, Pahor M, Manini T, et al. Increased inflammation but similar physical composition and function in older-aged, HIV-1 infected subjects. BMC Immunol. 2015;16:43.

7. Arts MH, Collard RM, Comijs HC, Naude PJ, Risselada R, Naarding P, Oude Voshaar RC. Relationship Between Physical Frailty and Low-Grade Inflammation in LateLife Depression. J Am Geriatr Soc. 2015;63(8):16521657.

8. Saum KU, Dieffenbach AK, Jansen EH, Schottker B, Holleczek B, Hauer K, Brenner H. Association between Oxidative Stress and Frailty in an Elderly German Population: Results from the ESTHER Cohort Study. Gerontology. 2015;61(5):407-415.

9. Hwang AC, Liu LK, Lee WJ, Chen LY, Peng LN, Lin MH, Chen LK. Association of Frailty and Cardiometabolic Risk Among Community-Dwelling Middle-Aged and Older People: Results from the I-Lan Longitudinal Aging Study. Rejuvenation Res. 2015;18(6):564-572.

10. Lealdini V, Trufelli DC, da Silva FB, Normando SR, Camargo EW, Matos LL, Fonseca FL, et al. Applicability of modified Glasgow Prognostic Score in the assessment of elderly patients with cancer: A pilot study. J Geriatr Oncol. 2015;6(6):479-483.

11. Aguirre LE, Jan IZ, Fowler K, Waters DL, Villareal DT, Armamento-Villareal R. Testosterone and Adipokines are Determinants of Physical Performance, Strength, and Aerobic Fitness in Frail, Obese, Older Adults. Int J Endocrinol. 2014;2014:507395.

12. Lai HY, Chang HT, Lee YL, Hwang SJ. Association between inflammatory markers and frailty in institutionalized older men. Maturitas. 2014;79(3):329-333.

13. Lin CC, Wu FY, Liao LN, Li CI, Lin CH, Yang CW, Meng $\mathrm{NH}$, et al. Association of CRP gene polymorphisms with serum CRP level and handgrip strength in community- 
dwelling elders in Taiwan: Taichung Community Health Study for Elders (TCHS-E). Exp Gerontol. 2014;57:141148.

14. Gale CR, Baylis D, Cooper C, Sayer AA. Inflammatory markers and incident frailty in men and women: the English Longitudinal Study of Ageing. Age (Dordr). 2013;35(6):2493-2501.

15. Beleigoli AM, Boersma E, Diniz Mde F, Vidigal PG, Lima-Costa MF, Ribeiro AL. C-reactive protein and Btype natriuretic peptide yield either a non-significant or a modest incremental value to traditional risk factors in predicting long-term overall mortality in older adults. PLoS One. 2013;8(9):e75809.

16. Baylis D, Bartlett DB, Syddall HE, Ntani G, Gale CR, Cooper C, Lord JM, et al. Immune-endocrine biomarkers as predictors of frailty and mortality: a 10-year longitudinal study in community-dwelling older people. Age (Dordr). 2013;35(3):963-971.

17. Almeida OP, Norman PE, van Bockxmeer FM, Hankey GJ, Flicker L. CRP 1846G $>$ A polymorphism increases risk of frailty. Maturitas. 2012;71(3):261-266.

18. Canon ME, Crimmins EM. Sex differences in the association between muscle quality, inflammatory markers, and cognitive decline. J Nutr Health Aging. 2011;15(8):695698.

19. Giovannini S, Onder G, Liperoti R, Russo A, Carter C, Capoluongo E, Pahor M, et al. Interleukin-6, C-reactive protein, and tumor necrosis factor-alpha as predictors of mortality in frail, community-living elderly individuals. J Am Geriatr Soc. 2011;59(9):1679-1685.

20. Wassel CL, Barrett-Connor E, Laughlin GA. Association of circulating C-reactive protein and interleukin-6 with longevity into the 80s and 90s: The Rancho Bernardo Study. J Clin Endocrinol Metab. 2010;95(10):4748-4755.

21. Tiainen K, Hurme M, Hervonen A, Luukkaala T, Jylha M. Inflammatory markers and physical performance among nonagenarians. J Gerontol A Biol Sci Med Sci. 2010;65(6):658-663.

22. Yoshida Y, Iwasa H, Kumagai S, Yoshida H, Suzuki T. Association between C-reactive protein (CRP) level and physical performance in community-dwelling elderly in Japan. Arch Gerontol Geriatr. 2010;51(2):164-168.

23. Wu IC, Shiesh SC, Kuo PH, Lin XZ. High oxidative stress is correlated with frailty in elderly chinese. J Am Geriatr Soc. 2009;57(9):1666-1671.

24. Reiner AP, Aragaki AK, Gray SL, Wactawski-Wende J, Cauley JA, Cochrane BB, Kooperberg CL, et al. Inflammation and thrombosis biomarkers and incident frailty in postmenopausal women. Am J Med. 2009;122(10):947954.

25. Hubbard RE, O'Mahony MS, Savva GM, Calver BL, Woodhouse KW. Inflammation and frailty measures in older people. J Cell Mol Med. 2009;13(9B):3103-3109.

26. Hubbard RE, O'Mahony MS, Calver BL, Woodhouse KW. Plasma esterases and inflammation in ageing and frailty. Eur J Clin Pharmacol. 2008;64(9):895-900.

27. Jylha M, Paavilainen P, Lehtimaki T, Goebeler S, Karhunen PJ, Hervonen A, Hurme M. Interleukin-1 receptor antagonist, interleukin-6, and C-reactive protein as predictors of mortality in nonagenarians: the vitality $90+$ study. J Gerontol A Biol Sci Med Sci. 2007;62(9):10161021.

28. Puts MT, Visser M, Twisk JW, Deeg DJ, Lips P. Endocrine and inflammatory markers as predictors of frailty. Clin Endocrinol (Oxf). 2005;63(4):403-411.

29. Cesari M, Penninx BW, Pahor M, Lauretani F, Corsi AM, Rhys Williams G, Guralnik JM, et al. Inflammatory markers and physical performance in older persons: the InCHIANTI study. J Gerontol A Biol Sci Med Sci. 2004;59(3):242-248.

30. Kojima G. Frailty as a predictor of disabilities among community-dwelling older people: a systematic review and meta-analysis. Disabil Rehabil. 2016:1-12.

31. Poli S, Cella A, Puntoni M, Musacchio C, Pomata M, Torriglia D, Vello N, et al. Frailty is associated with socioeconomic and lifestyle factors in community-dwelling older subjects. Aging Clin Exp Res. 2016.

32. Fontana L, Addante F, Copetti M, Paroni G, Fontana A, Sancarlo D, Pellegrini F, et al. Identification of a metabolic signature for multidimensional impairment and mortality risk in hospitalized older patients. Aging Cell. 2013;12(3):459-466.

33. Topinkova E. Aging, disability and frailty. Ann Nutr Metab. 2008;52(Suppl 1):6-11.

34. Hamerman D. Toward an understanding of frailty. Ann Intern Med. 1999;130(11):945-950.

35. Morley JE, Baumgartner RN. Cytokine-related aging process. J Gerontol A Biol Sci Med. 2004;59:924-929.

36. Ferrucci L, Harris TB, Guralnik JM, Tracy RP, Corti MC, Cohen HJ, Penninx B, et al. Serum IL-6 level and the development of disability in older persons. J Am Geriatr Soc. 1999;47(6):639-646.

37. Li H, Manwani B, Leng SX. Frailty, inflammation, and immunity. Aging Dis. 2011;2(6):466-473.

38. Boxer RS, Dauser DA, Walsh SJ, Hager WD, Kenny AM. The association between vitamin $\mathrm{D}$ and inflammation with the 6-minute walk and frailty in patients with heart failure. J Am Geriatr Soc. 2008;56(3):454-461.

39. Collerton J, Martin-Ruiz C, Davies K, Hilkens CM, Isaacs J, Kolenda C, Parker C, et al. Frailty and the role of inflammation, immunosenescence and cellular ageing in the very old: cross-sectional findings from the Newcastle 85+ Study. Mech Ageing Dev. 2012;133(6):456-466.

40. Bourdel-Marchasson I, Laksir H, Puget E. Interpreting routine biochemistry in those aged over 65 years: a time for change. Maturitas. 2010;66(1):39-45.

41. Yano Y, Matsuda S, Hatakeyama K, Sato Y, Imamura T, Shimada K, Kodama T, et al. Plasma Pentraxin 3, but not high-sensitivity C-reactive protein, is a useful inflammatory biomarker for predicting cognitive impairment in elderly hypertensive patients. J Gerontol A Biol Sci Med Sci. 2010;65(5):547-552.

42. Walston J, McBurnie MA, Newman A, Tracy RP, Kop WJ, Hirsch $\mathrm{CH}$, Gottdiener J, et al. Frailty and activation of the inflammation and coagulation systems with and without clinical comorbidities: results from the Cardiovascular Health Study. Arch Intern Med. 2002;162(20):23332341. 